\title{
A Comprehensive Model to Predict Severe Acute Graft-Versus-Host Disease after Haploidentical Hematopoietic Stem Cell Transplantation
}

\section{Meng-Zhu Shen}

Peking University People's Hospital, Peking University Institute of Hematology, National Clinical

Research Center for Hematologic Disease

\section{Shen-Da Hong}

National Institute of Health Data Science at Peking University, Peking University Health Science Center

\section{Rui Lou}

Peking University People's Hospital, Peking University Institute of Hematology, National Clinical

Research Center for Hematologic Disease

\section{Rui-Ze Chen}

The First Affiliated Hospital of Nanjing Medical University

\section{Xiao-Hui Zhang}

Peking University People's Hospital, Peking University Institute of Hematology, National Clinical Research Center for Hematologic Disease

\section{Lan-Ping Xu}

Peking University People's Hospital, Peking University Institute of Hematology, National Clinical Research Center for Hematologic Disease

\section{Yu Wang}

Peking University People's Hospital, Peking University Institute of Hematology, National Clinical Research Center for Hematologic Disease

\section{Chen-Hua Yan}

Peking University People's Hospital, Peking University Institute of Hematology, National Clinical Research Center for Hematologic Disease

\section{Huan Chen}

Peking University People's Hospital, Peking University Institute of Hematology, National Clinical Research Center for Hematologic Disease

\section{Yu-Hong Chen}

Peking University People's Hospital, Peking University Institute of Hematology, National Clinical Research Center for Hematologic Disease

\section{Wei Han}

Peking University People's Hospital, Peking University Institute of Hematology, National Clinical Research Center for Hematologic Disease 


\section{Feng-Rong Wang}

Peking University People's Hospital, Peking University Institute of Hematology, National Clinical Research Center for Hematologic Disease

\section{Jing-Zhi Wang}

Peking University People's Hospital, Peking University Institute of Hematology, National Clinical Research Center for Hematologic Disease

\section{Kai-Yan Liu}

Peking University People's Hospital, Peking University Institute of Hematology, National Clinical Research Center for Hematologic Disease

\section{Xiao-Jun Huang}

Peking University People's Hospital, Peking University Institute of Hematology, National Clinical Research Center for Hematologic Disease

\section{Xiao-Dong Mo ( $\nabla$ mxd453@163.com )}

Peking University People's Hospital, Peking University Institute of Hematology, National Clinical Research Center for Hematologic Disease

\section{Research Article}

Keywords: acute leukemia, acute graft-versus-host disease, haploidentical donor, hematopoietic stem cell transplant, predicted model

Posted Date: February 11th, 2022

DOI: https://doi.org/10.21203/rs.3.rs-1335388/v1

License: (c) (1) This work is licensed under a Creative Commons Attribution 4.0 International License. Read Full License 


\section{Abstract}

Background: Acute graft-versus-host disease (aGVHD) remains the major cause of early mortality after haploidentical related donor (HID) hematopoietic stem cell transplantation (HSCT). We aimed to establish a comprehensive model which could predict severe aGVHD after HID HSCT.

Methods: Consecutive 470 acute leukemia patients receiving HID HSCT according to the protocol registered at https://clinicaltrials.gov (NCT03756675) were enrolled, 70\% of them $(n=335)$ were randomly selected as training cohort and the remains $30 \%(n=135)$ were used as validation cohort.

Results: The equation was as follows: Probability (grade III-IV aGVHD) $=1 / 1+\exp (-\mathrm{Y})$, where $\mathrm{Y}=-0.0288$ $\times($ age $)+0.7965 \times($ gender $)+0.8371 \times(C D 3+/$ CD14 + cells ratio in graft $)+0.5829 \times($ donor $/$ recipient relation $)-0.0089 \times(C D 8+$ cell counts in graft $)-2.9046$. The threshold of probability was 0.057392 which helped separate patients into high- and low-risk groups. The 100-day cumulative incidence of grade III-IV aGVHD in the low- and high-risk groups was $4.1 \%(95 \% \mathrm{Cl}, 1.9 \%-6.3 \%)$ versus $12.8 \%(95 \% \mathrm{Cl}$, $7.4 \%-18.2 \%)(P=0.001), 3.2 \%(95 \% \mathrm{Cl}, 1.2 \%-5.1 \%)$ versus $10.6 \%(95 \% \mathrm{Cl}, 4.7 \%-16.5 \%)(P=0.006)$, and $6.1 \%(95 \% \mathrm{Cl}, 1.3 \%-10.9 \%)$ versus $19.4 \%(95 \% \mathrm{Cl}, 6.3 \%-32.5 \%)(P=0.017)$, respectively, in total, training, and validation cohort. The rates of grade III-IV skin and gut aGVHD in high-risk group were both significantly higher than those of low-risk group. This model could also predict grade II-IV and grade I-IV aGVHD.

Conclusions: We established a model which could predict the development of severe aGVHD in HID HSCT recipients.

\section{Introduction}

Allogeneic hematopoietic stem cell transplantation (allo-HSCT) is the most important curative method for acute leukemia (AL), which can significantly improve the long-term survival [1, 2]. Human leukocyte antigen (HLA) haploidentical related donors (HIDs) have become one of the most important donors, which accounted for the proportion at $42 \%$ among allo-HSCT from family donors in Europe [3], and accounted for the proportion at $60 \%$ among all of the allo-HSCT in China [4].

Although many strategies (e.g., antithymocyte globulin [ATG] and post-transplant cyclophosphamide [PTCy]) are used to prevent acute graft-versus-host disease (aGVHD), it is still inevitable [5]. Only half of aGVHD patients could achieve durable responses to initial corticosteroid therapy [6], and there is no standard therapy for steroid refractory aGVHD and the survival among these patients is poor [7]. Thus, severe aGVHD remains the major cause of early mortality after HID HSCT [8-10]. An early-warning method for severe aGVHD can help to provide risk-stratification directed prophylaxis for aGVHD and significantly improve the survival of patients receiving HID HSCT.

Several demographic and transplant characteristics, such as patient age, underlying disease (e.g., chronic myeloid leukemia), comorbidities before allo-HSCT, donor/recipient gender mismatching (i.e., female 
donor/male recipient combination), donor and recipient cytomegalovirus (CMV) serostatus, donor type (i.e., HLA-non-identical donors), HLA disparity, and GVHD prophylaxis methods are reported as important risk factors for aGVHD [11, 12]. Particularly, donor/recipient relation (i.e., collateral relative donors [CRDs] and maternal donors [MDs]) is associated with aGVHD after HID HSCT $[13,14]$.

In addition, graft composition may be associated with aGVHD after allo-HSCT. For example, CD34+ cell counts [15], CD3+ cell counts [15], CD8+ cell counts [16], and CD19+ cell counts [17] contained in a graft could predict aGVHD after identical sibling donor (ISDs) or unrelated donor (URDs) HSCT. Particularly, the CD4+/CD8+ T cells ratio in granulocyte colony-stimulating factor (G-CSF)-mobilized bone marrow (GBM) [18] or the CD3+/CD14+ cells ratio in G-CSF-primed peripheral blood (G-PB) [19] can predict aGVHD after HID HSCT. However, most of the studies only reported the risk factors for aGVHD, and there was no comprehensive model which included the characteristics of demographic, disease, transplant, and graft composition for aGVHD prediction.

Thus, in the present study, we aimed to establish a comprehensive model which could predict the severe aGVHD after HID HSCT.

\section{Patients And Methods}

\subsection{Study Design}

Consecutive AL patients receiving HID HSCT between January 21, 2020 and May 31, 2021 at Peking University, Institute of Hematology (PUIH) were enrolled. The end point of the last follow-up for all survivors was November 11, 2021. A total of 67 patients had been previously reported by Ma et al.[20], and all of them were further followed-up. All patients were treated according to the protocol registered at https://clinicaltrials.gov (NCT03756675). Informed consent was obtained from all patients or their guardians. The study was conducted in accordance with the Declaration of Helsinki, and the protocol was approved by the Institutional Review Board of Peking University People's Hospital.

\subsection{Transplant regimens}

Major conditioning regimen consisted of cytarabine, busulfan, cyclophosphamide, and semustine [21, 22]. Twelve patients received total body irradiation (TBI)-based conditioning regimen. G-PB harvests were administered to the recipients on the same day of collection [20]. ATG, cyclosporine A, mycophenolate mofetil, and short-term methotrexate were administered to prevent GVHD. Particularly, patients with CRDs or MDs could receive low dose cyclophosphamide after transplantation based on ATG for GVHD prophylaxis (Supplementary methods) [23].

\subsection{Evaluation of graft composition}

The methods for graft composition evaluation were showed in Supplementary methods [18, 24].

\subsection{Definitions}


The definitions for disease risk index (DRI), engraftment, aGVHD, relapse, mortality, and survival were showed in Supplementary methods [25-27].

\subsection{Building machine learning models}

Our method consisted of three steps: selecting features, building models, and finding the optimal threshold (Fig. 1 and Supplementary methods)

\subsubsection{Backward feature selection strategy}

We randomly selected $70 \%$ of the entire population $(n=335)$ as the training cohort, the remains $30 \%$ were used as validation cohort $(n=135)$. For primary outcome (i.e., grade III-IV aGVHD), the model building steps were performed in the training cohort and validated in the validation cohort. The sensitivity, specificity, area under curve score, and accuracy score were identified in both the training and validation cohort.

We used feature selection techniques to select the predictive variables (Supplementary methods) [28]. By doing this, we could reduce the complexity of machine learning model, while also improve the generalizability. We set age and gender to be obligate variables in the machine learning model. For other variables, we selected top-3 significant variables using backward feature selection strategy. In detail, we started with all variables including age and gender. At each iteration, we removed the least significant variable (variable with the highest p-value) except age and gender. Aside from the involved variables, we also added an extra constant variate to make the feature selection more robust. The selection was realized using generalized linear models with binomial exponential family distribution of statsmodels v0.13.0 statistical models module with Python 3.8 based on anaconda3 development platform [29].

\subsubsection{Building models}

We used generalized linear models with binomial exponential family distribution to realize logistic regression models, which were equivalent models. Aside from the selected variables, we added an extra constant variate for the predicted model to make the machine learning models stronger. We used statsmodels v0.13.0 with Python 3.8 to build the models based on anaconda3 development platform. The model parameters were set to be the defaults [30-32].

\subsubsection{Finding the optimal threshold}

Logistic regression model produced values between 0 and 1, which could be treated as the probabilities to be positive prediction. We needed to determine the threshold of output positive predictions (1) or negative predictions (0). In detail, we drew Receiver Operating Characteristic (ROC) curves [33] and calculated the g-mean for each threshold [34]. The best threshold corresponded to the largest g-mean. The g-mean was calculated as sqrt(tprx (1-fpr)), where tpr represented true positive rate, fpr represented false positive rate, under a given threshold. 


\subsubsection{Evaluation for model}

ROC-AUC was defined as the area under the curve of the true positive rate versus the false positive rate at various thresholds ranging from zero to one. Confusion matrix was a summary table of predictions. In this paper, the confusion matrix was of two-by-two shape. The diagonal showed the count values of correct predictions, while the others showed the count values of incorrect predictions. Besides, we also normalized the count values by the number of True Label (Outcome) or the number of Predicted Label (Prediction). To better visualize the matrix, we colored the values with Blues colorbar.

\subsection{Statistical methods}

In the present study, the primary outcome was grade III to IV aGVHD. The secondary outcomes included grade II to IV aGVHD, grade I to IV aGVHD, relapse, non-relapse mortality (NRM), leukemia-free survival (LFS), and overall survival (OS).

Mann-Whitney U-test was used to compare continuous variables, $\chi^{2}$ and Fisher's exact tests were used for categorical variables. The Kaplan-Meier method was used to estimate the probability of LFS and OS. Competing risk analyses were performed to calculate the cumulative incidence of aGVHD, relapse, and NRM [35]. Testing was two-sided at the $P<0.05$ level. Statistical analysis was performed on SPSS 22.0 software (SPSS, Chicago, IL), and R software (version 4.0.0) (http://www.r-project.org).

\section{Results}

\subsection{Patient characteristics}

A total of 470 patient were enrolled, and the characteristics were all comparable between training and validation cohort (Table 1). All patients achieved neutrophil engraftment and the median time from HSCT to neutrophil engraftment was 12 days (range, 9-28) days. Four hundred and fifty-eight (97.4\%) patients achieved platelet engraftment and the median time from HSCT to platelet engraftment was 13 days (range, 7-144) days, respectively.

Two hundred and sixty-six (56.6\%), 129 (27.4\%), and 33 (7.0\%) patients experienced grade I to IV aGVHD, grade II to IV aGVHD, and grade III to IV aGVHD after allo-HSCT, respectively. The median time from HSCT to aGVHD was 20 days (range, 8-99) days. The cumulative incidence of grade I to IV aGVHD, grade II to IV aGVHD, and grade III to IV aGVHD at 100 days after HID HSCT was 56.5\% (95\% Cl, 52.0\%-61.0\%), $27.3 \%(95 \% \mathrm{Cl}, 23.3 \%-31.3 \%)$, and $6.8 \%(95 \% \mathrm{Cl}, 4.5 \%-9.1 \%)$, respectively.

Thirty-eight (8.1\%) patients experienced relapse, and 16 (3.4\%) patients died of NRM. Four hundred and forty-nine patients survived until the last follow-up, and the median duration of follow-up was 200 days (range, 52 to 509) days. The probabilities of relapse, NRM, LFS, and OS at 100 days after HID HSCT were $2.8 \%(95 \% \mathrm{Cl}, 1.3 \%-4.3 \%), 1.5 \%(95 \% \mathrm{Cl}, 0.4 \%-2.6 \%), 95.7 \%(95 \% \mathrm{Cl}, 93.9 \%-97.6 \%)$, and $97.8 \%(95 \% \mathrm{Cl}$, $96.5 \%-99.2 \%)$, respectively. 


\subsection{Predicted model for grade III to IV aGVHD (model 1)}

A predictive model for grade III-IV aGVHD was developed (Supplementary methods, Table S1 and Fig. S1), and the equation was as follows:

Probability (grade III-IV aGVHD) $=\frac{1}{1+\exp (-\mathrm{Y})}$

where

$Y=-0.0288 \times($ age $)+0.7965 \times($ gender $)+0.8371 \times(C D 3+/$ CD14+ cells ratio in graft $)+0.5829 \times$ (donor/recipient relation) $-0.0089 \times($ CD8+ cell counts in graft $)-2.9046$. Particularly, donor/recipient relation included immediate relative donors other than MDs (value=0), MDs (value=1), and CRDs (value=2). Gender included male (value=0) and female (value=1). The age (years), CD8+ cell counts $\left(\times 10^{6} / \mathrm{kg}\right), \mathrm{CD} 3+/ \mathrm{CD} 14+$ cells ratio in graft used actual numerical value (Table S1). The threshold of probability was 0.057392 and the g-mean was 0.682. Patients were separated into low- and high-risk groups by the threshold.

In the training cohort, the sensitivity, specificity, area under curve score, and accuracy score were 0.632 , $0.680,0.685$, and 0.678 , respectively. ROC curve for the model and confusion matrix is shown in Fig.

2A and Table S2. In the validation cohort, the sensitivity, specificity, area under curve score, and accuracy score were $0.500,0.760,0.673$, and 0.733 , respectively. ROC curve for the model and confusion matrix is shown in Fig. 2B and Table S3.

\subsection{Verifying the predicted model in validation and total cohort}

The 100-day cumulative incidence of grade III-IV aGVHD in the low- and high-risk groups was $4.1 \%$ $(95 \% \mathrm{Cl}, 1.9 \%-6.3 \%)$ versus $12.8 \%(95 \% \mathrm{Cl}, 7.4 \%-18.2 \%)(P=0.001)$, respectively, in total cohort (Fig. 3A).

The 100-day cumulative incidence of grade III-IV aGVHD in the low- and high-risk groups was 3.2\% $(95 \% \mathrm{Cl}, 1.2 \%-5.1 \%)$ versus $10.6 \%(95 \% \mathrm{Cl}, 4.7 \%-16.5 \%)$ with $P=0.006$ and $6.1 \%(95 \% \mathrm{Cl}, 1.3 \%-10.9 \%)$ versus $19.4 \%(95 \% \mathrm{Cl}, 6.3 \%-32.5 \%)$ with $P=0.017$, respectively, in training cohort (Fig. 3B) and validation cohort (Fig. 3C).

The rates of grade III to IV skin and gut aGVHD in low-risk group were both significantly lower than those of high-risk group (skin: $4.4 \%$ vs. $12.8 \%, P=0.001$; gut: $1.6 \%$ vs. $4.7 \%, P=0.045$ ) (Fig. 3D).

\subsection{Validation of the predicted model in grade II to IV aGVHD}

In the total population, the 100-day cumulative incidence of grade II to IV aGVHD in the low-risk group and high-risk group was $21.5 \%(95 \% \mathrm{Cl}, 17.0 \%-26.0 \%)$ and $39.6 \%(95 \% \mathrm{Cl}, 31.7 \%-47.5 \%)$, respectively $(P<$ 0.001 , Fig. 4A). The rates of grade II to IV skin and gut aGVHD in the low-risk group were both 
significantly lower than those of high-risk group (skin: $25.5 \%$ vs. $35.6 \%, P=0.025$; gut: $7.5 \%$ vs. $18.8 \%, P<$ 0.001) (Fig. 4B).

\subsection{Validation of the predicted model in grade I to IV aGVHD}

In total population, the 100-day cumulative incidence of grade I to IV aGVHD in the low-risk group and high-risk group was $51.5 \%(95 \% \mathrm{Cl}, 46.0 \%-57.0 \%)$ and $67.1 \%(95 \% \mathrm{Cl}, 59.5 \%-74.7 \%)$, respectively $(P=$ 0.001 , Fig. 4C). The rates of grade I to IV skin, gut, and liver aGVHD in the low-risk group were all significantly lower than those of high-risk group (skin: $44.5 \%$ vs. $60.4 \%, P=0.001$; gut: $15.9 \%$ vs. $30.2 \%$, $P<0.001$; liver: $1.9 \%$ vs. $5.4 \%, P=0.038$ ) (Fig. 4D).

\subsection{Validation of the predicted model in other clinical outcomes after HSCT}

In total population, the probabilities of relapse, NRM, LFS, and OS at 100 days after HID HSCT were all comparable between the low- and high-risk groups in the total population (Fig. S2A-D).

\section{Discussion}

In the present study, we established a predicted model for grade III to IV aGVHD including patient age, gender, donor/recipient relation, CD8+ T cell count, and CD3+/CD14+ cells ratio in the graft in training cohort, which was verified in validation and total cohorts. To the best of our knowledge, we firstly established a comprehensive model which can effectively predict severe aGVHD in allo-HSCT recipients.

Donor/recipient relation is an important risk factor for aGVHD after HID HSCT [36]. Wang et al. [13] reported that MDs showed the higher risk of aGVHD among immediate relative donors. Mo et al. [14] reported that the risk of aGVHD was comparable between CRDs and MDs groups, which was significantly higher than the paternal donors group. Wang et al.[23] reported that low-dose PTCy combined with ATG could help to decrease incidence of grade III to IV aGVHD in HID HSCT recipients with CRDs and MDs, which was comparable with HID HSCT recipients with immediate relative donor other than MDs [37]. However, these studies included patients with myelodysplastic syndromes and chronic myeloid leukemia which were different from the present study. We observed that in the disease-specific population of patients with AL, although some patients receiving low-dose PTCy based on ATG for GVHD prophylaxis, CRDs and MDs were still associated with the development of severe aGVHD.

We observed that $C D 3+/ C D 14+$ cells ratio in the graft was another important variable in the model. $T$ cell plays a critical role in the pathogenesis of aGVHD [38], and depleting T lymphocytes could significantly reduce the risk of GVHD [39, 40]. Czerw et al. [15] reported that a higher CD3+ cells count in the graft was associated with the increased risk of aGVHD. On the other hand, the count of CD14+ cells which mainly presented the monocyte in the graft might also be associated with aGVHD [41]. Similarly, Liu et al. [19] also observed that CD3+/CD14+ cells ratio in G-PB could predict aGVHD in patients receiving HID HSCT. 
The counts of CD8+ T cells in the graft was also included in the predicted model. CD8+ T cells were thought critical for aGVHD pathogenesis $[38,42,43]$. CD8+ $T$ cells were more abundant than CD4+ $T$ cells in the blood of mice after aGVHD induction, and the severity of aGVHD was associated with the infiltration of CD8+ T cells in target tissues [44]. Several studies reported that CD8+ T cells count in the graft could predict the aGVHD in patient receiving ISD HSCT [16], URD HSCT [45], and umbilical cord blood transplantation [46].

We observed that our predict model was associated with grade III to IV and grade II to IV gut aGVHD after HID HSCT, which suggested that routine GVHD prophylaxis methods were not sufficient to prevent severe gut aGVHD in high-risk patients. Severe gut aGVHD is difficult to treat and is the greatest cause of GVHDrelated mortality [47]. Thus, our predicted model could help to direct more intense prophylaxis for gut aGVHD in high-risk patients.

Our comprehensive model could predict severe aGVHD, however, it was not associated with 100-day mortality and survival. Several researches observed a significantly higher incidence of NRM and a lower incidence of OS and LFS in patients with severe aGVHD [48-52]. In contrast, the 100-day cumulative incidence of NRM after HID HSCT was only $1.5 \%$ in the present study. This may be because PUIH is the largest transplant center in China and has rich experience in GVHD therapy [53-55]. Thus, GVHD related death was not the leading cause of death in PUIH [56]. This could not represent the level of GVHD therapies in other transplant centers, for example, Deng et al. [57] reported that the mortality of grade III to IV aGVHD patients was as high as $81.0 \%$ in their hospital in China. In addition, it could not alter the fact that patients with severe aGVHD would experience great pain and economic burden.

The present study had some limitations. Firstly, the model was not associated with the development of grade III to IV liver aGVHD after HID HSCT, which might be due to the small sample of severe liver aGVHD in the present study. However, we observed that the rate of grade I to IV liver aGVHD in high-risk group was higher than that of low-risk group. Secondly, although we verified the model successfully in the validation cohort, this was a single-center study and the sample of validation cohort was relatively small. Thus, the model should be further evaluated by independent cohorts in multicenter studies. Lastly, we did not monitor plasma cytokines (e.g., interleukin [IL]-2) and biomarkers (e.g., ST2, REG3a, TNFR1, and IL$2 \mathrm{Ra})[58,59]$, which may further improve the efficacy of our predicted model.

\section{Conclusions}

We established a comprehensive model which could predict the development of severe aGVHD in HID HSCT recipients. This was the first predicted model for severe aGVHD which can be popularized easily, can help to provide risk-stratification directed aGVHD prophylaxis, and may further decrease the risk of severe aGVHD in HID HSCT recipients. In future, prospective, multicenter studies can further confirm the efficacy of our predicted model.

\section{Declarations}




\section{Ethics approval}

The study was conducted in accordance with the Declaration of Helsinki, and the protocol was approved by the Institutional Review Board of Peking University People's Hospital.

\section{Consent to Participate}

Informed consent was obtained from all individual participants or their guardians included in the study.

\section{Consent to Publish}

Not applicable.

\section{Availability of data and materials}

The datasets generated during the analysis of the current study are available from the corresponding author on reasonable request.

\section{Competing Interests}

The authors have no relevant financial or non-financial interests to disclose.

\section{Funding}

This work was supported by the Program of the National Natural Science Foundation of China (grant number 82170208), the Foundation for Innovative Research Groups of the National Natural Science Foundation of China (grant number 81621001), the CAMS Innovation Fund for Medical Sciences (CIFMS) (grant number 2019-I2M-5-034), the Key Program of the National Natural Science Foundation of China (grant number 81930004), and the Fundamental Research Funds for the Central Universities.

\section{Authors Contributions}

Meng-Zhu Shen: Investigation; Software; Visualization; Writing - original draft; Writing - review \& editing.

Shen-Da Hong: Software; Visualization; Writing - original draft.

Rui Lou: Data curation; Resources; Formal analysis.

Rui-Ze Chen: Data curation; Resources; Formal analysis.

Xiao-Hui Zhang: Data curation; Resources.

Lan-Ping Xu: Data curation; Resources.

Yu Wang: Data curation; Resources.

Chen-Hua Yan: Data curation; Resources. 
Huan Chen: Data curation; Resources.

Yu-Hong Chen: Data curation; Resources.

Wei Han: Data curation; Resources.

Feng-Rong Wang: Data curation; Resources.

Jing-Zhi Wang: Data curation; Resources.

Kai-Yan Liu: Data curation; Resources.

Xiao-Jun Huang: Conceptualization; Funding acquisition; Investigation; Methodology; Project administration; Data curation; Resources; Supervision; Validation; Visualization.

Xiao-Dong Mo: Conceptualization; Data curation; Formal analysis; Investigation; Methodology; Project administration; Resources; Software; Supervision; Validation; Visualization; Writing -original draft; Writing - review \& editing.

\section{Acknowledgements:}

Not applicable.

\section{References}

1. Zhang XH, Chen J, Han MZ, Huang H, Jiang EL, Jiang M, et al. The consensus from The Chinese Society of Hematology on indications, conditioning regimens and donor selection for allogeneic hematopoietic stem cell transplantation: 2021 update. J Hematol Oncol. 2021;14(1):145.

2. Xu L, Chen H, Chen J, Han M, Huang H, Lai Y, et al. The consensus on indications, conditioning regimen, and donor selection of allogeneic hematopoietic cell transplantation for hematological diseases in China-recommendations from the Chinese Society of Hematology. J Hematol Oncol. 2018;11(1):33.

3. Passweg JR, Baldomero H, Chabannon C, Basak GW, de la Cámara R, Corbacioglu S, et al. Hematopoietic cell transplantation and cellular therapy survey of the EBMT: monitoring of activities and trends over 30 years. Bone Marrow Transplant. 2021;56(7):1651-64.

4. Xu LP, Lu PH, Wu DP, Sun ZM, Liu QF, Han MZ, et al. Hematopoietic stem cell transplantation activity in China 2019: a report from the Chinese Blood and Marrow Transplantation Registry Group. Bone Marrow Transplant. 2021:1-8.

5. Ringdén $O$, Labopin M, Sadeghi B, Mailhol A, Beelen D, Fløisand Y, et al. What is the outcome in patients with acute leukaemia who survive severe acute graft-versus-host disease? J Intern Med. 2018;283(2):166-77. 
6. Martin PJ, Rizzo JD, Wingard JR, Ballen K, Curtin PT, Cutler C, et al. First- and second-line systemic treatment of acute graft-versus-host disease: recommendations of the American Society of Blood and Marrow Transplantation. Biol Blood Marrow Transplant. 2012;18(8):1150-63.

7. Penack $O$, Marchetti M, Ruutu T, Aljurf M, Bacigalupo A, Bonifazi F, et al. Prophylaxis and management of graft versus host disease after stem-cell transplantation for haematological malignancies: updated consensus recommendations of the European Society for Blood and Marrow Transplantation. Lancet Haematol. 2020;7(2):e157-e67.

8. Yeshurun M, Weisdorf D, Rowe JM, Tallman MS, Zhang MJ, Wang HL, et al. The impact of the graftversus-leukemia effect on survival in acute lymphoblastic leukemia. Blood Adv. 2019;3(4):670-80.

9. Yu J, Parasuraman S, Shah A, Weisdorf D. Mortality, length of stay and costs associated with acute graft-versus-host disease during hospitalization for allogeneic hematopoietic stem cell transplantation. Curr Med Res Opin. 2019;35(6):983-8.

10. Modi A, Rybicki L, Majhail NS, Mossad SB. Severity of acute gastrointestinal graft-vs-host disease is associated with incidence of bloodstream infection after adult allogeneic hematopoietic stem cell transplantation. Transpl Infect Dis. 2020;22(1):e13217.

11. Blume KG, Thomas ED. Thomas' Hematopoietic Cell Transplantation. Fifth Edition ed. Zaia JA, editor: John Wiley \& Sons, Ltd; 2016.

12. Maziarz R, Slater S. Blood and Marrow Transplant Handbook Comprehensive Guide for Patient Care: Comprehensive Guide for Patient Care2021.

13. Wang Y, Chang YJ, Xu LP, Liu KY, Liu DH, Zhang XH, et al. Who is the best donor for a related HLA haplotype-mismatched transplant? Blood. 2014;124(6):843-50.

14. Mo XD, Zhang YY, Zhang XH, Xu LP, Wang Y, Yan $C H$, et al. The role of collateral related donors in haploidentical hematopoietic stem cell transplantation. Sci Bull. 2018;63(20):1376-82.

15. Czerw T, Labopin M, Schmid C, Cornelissen JJ, Chevallier P, Blaise D, et al. High CD3+ and CD34+ peripheral blood stem cell grafts content is associated with increased risk of graft-versus-host disease without beneficial effect on disease control after reduced-intensity conditioning allogeneic transplantation from matched unrelated donors for acute myeloid leukemia - an analysis from the Acute Leukemia Working Party of the European Society for Blood and Marrow Transplantation. Oncotarget. 2016;7(19):27255-66.

16. Mohty M, Bagattini S, Chabannon C, Faucher C, Bardou VJ, Bilger K, et al. CD8+ T cell dose affects development of acute graft-vs-host disease following reduced-intensity conditioning allogeneic peripheral blood stem cell transplantation. Exp Hematol. 2004;32(11):1097-102.

17. Svenberg $P$, Wang $T$, Uhlin $M$, Watz E, Remberger $M$, Ringden $O$, et al. The importance of graft cell composition in outcome after allogeneic stem cell transplantation in patients with malignant disease. Clin Transplant. 2019;33(6):e13537.

18. Luo XH, Chang YJ, Xu LP, Liu DH, Liu KY, Huang XJ. The impact of graft composition on clinical outcomes in unmanipulated HLA-mismatched/haploidentical hematopoietic SCT. Bone Marrow Transplant. 2009;43(1):29-36. 
19. Liu DH, Zhao XS, Chang YJ, Liu YK, Xu LP, Chen H, et al. The impact of graft composition on clinical outcomes in pediatric patients undergoing unmanipulated HLA-mismatched/haploidentical hematopoietic stem cell transplantation. Pediatr Blood Cancer. 2011;57(1):135-41.

20. Ma YR, Zhang X, Xu L, Wang Y, Yan C, Chen H, et al. G-CSF-Primed Peripheral Blood Stem Cell Haploidentical Transplantation Could Achieve Satisfactory Clinical Outcomes for Acute Leukemia Patients in the First Complete Remission: A Registered Study. Front Oncol. 2021;11:631625.

21. Wang Y, Liu QF, Lin R, Yang T, Huang XJ. Optimizing antithymocyte globulin dosing in haploidentical hematopoietic cell transplantation: Iong-term follow-up of a multicenter, randomized controlled trial. Sci Bull. 2021; 66(24): 2498-2505.

22. Wang Y, Liu QF, Xu LP, Liu KY, Zhang XH, Ma X, et al. Haploidentical vs identical-sibling transplant for AML in remission: a multicenter, prospective study. Blood. 2015;125(25):3956-62.

23. Wang Y, Wu DP, Liu QF, Xu LP, Liu KY, Zhang XH, et al. Low-dose post-transplant cyclophosphamide and anti-thymocyte globulin as an effective strategy for GVHD prevention in haploidentical patients. J Hematol Oncol. 2019;12(1):88.

24. Liu Y, Chen S, Yu H. Standardization and Quality Control in Flow Cytometric Enumeration of CD34(+) cells. Zhongguo Shi Yan Xue Ye Xue Za Zhi. 2000;8(4):302-6.

25. Armand P, Kim HT, Logan BR, Wang Z, Alyea EP, Kalaycio ME, et al. Validation and refinement of the Disease Risk Index for allogeneic stem cell transplantation. Blood. 2014;123(23):3664-71.

26. Mo XD, Zhang XH, Xu LP, Wang Y, Yan CH, Chen H, et al. Disease Risk Comorbidity Index for Patients Receiving Haploidentical Allogeneic Hematopoietic Transplantation. Engineering. 2021;7(2):162-9.

27. Harris AC, Young R, Devine S, Hogan WJ, Ayuk F, Bunworasate U, et al. International, Multicenter Standardization of Acute Graft-versus-Host Disease Clinical Data Collection: A Report from the Mount Sinai Acute GVHD International Consortium. Biol Blood Marrow Transplant. 2016;22(1):4-10.

28. Guyon I, Andre E. An introduction to variable and feature selection2003.

29. Nelder JA, Wedderburn RWM. Generalized Linear Models. Journal of the Royal Statistical Society: Series A (General). 1972;135(3):370-84.

30. Hosmer DWJ, Lemeshow SL. Applied Logistic Regression: Hoboken, New Jersey, USA: John Wiley and Sons, Inc; 1989 (398).

31. Seabold S, Perktold J. Statsmodels: Econometric and Statistical Modeling with Python: Proceedings of the 9th Python in Science Conference; 2010 (57): 61.

32. Hastie T. The Elements of Statistical Learning: Data Mining, Inference, and Prediction2009.

33. Zweig MH, Campbell G. Receiver-operating characteristic (ROC) plots: a fundamental evaluation tool in clinical medicine. Clinical Chemistry. 1993;39(4):561-77.

34. Guo H, Liu H, Wu C, Zhi W, Xiao Y, She W. Logistic discrimination based on G-mean and F-measure for imbalanced problem. J INTELL FUZZY SYST. 2016;31(3):1155-66.

35. Gooley TA, Leisenring W, Crowley J, Storer BE. Estimation of failure probabilities in the presence of competing risks: new representations of old estimators. Stat Med. 1999;18(6):695-706. 
36. Chang YJ, Luznik L, Fuchs EJ, Huang XJ. How do we choose the best donor for T-cell-replete, HLAhaploidentical transplantation? J Hematol Oncol. 2016;9:35.

37. Wang Y, Chang YJ, Chen L, Xu LP, Bian ZL, Zhang XH, et al. Low-dose post-transplant cyclophosphamide can mitigate GVHD and enhance the G-CSF/ATG induced GVHD protective activity and improve haploidentical transplant outcomes. Oncoimmunology. 2017;6(11):e1356152.

38. Zeiser R, Blazar BR. Acute Graft-versus-Host Disease - Biologic Process, Prevention, and Therapy. N Engl J Med. 2017;377(22):2167-79.

39. Boehmer H, Sprent J, Nabholz M. Tolerance to histocompatibility determinants in tetraparental bone marrow chimeras. J Exp Med. 1975;141(2):322-34.

40. Bortin MM, Saltzstein EC. Graft versus host inhibition: fetal liver and thymus cells to minimize secondary disease. Science. 1969;164(3877):316-8.

41. Wen Q, Zhao HY, Yao WL, Zhang YY, Fu HX, Wang Y, et al. Monocyte subsets in bone marrow grafts may contribute to a low incidence of acute graft-vs-host disease for young donors. J Cell Mol Med. 2020;24(16):9204-16.

42. Shlomchik WD. Graft-versus-host disease. Nat Rev Immunol. 2007;7(5):340-52.

43. Blazar BR, Murphy WJ, Abedi M. Advances in graft-versus-host disease biology and therapy. Nat Rev Immunol. 2012;12(6):443-58.

44. Theiss-Suennemann J, Jörß K, Messmann JJ, Reichardt SD, Montes-Cobos E, Lühder F, et al. Glucocorticoids attenuate acute graft-versus-host disease by suppressing the cytotoxic capacity of CD8(+) T cells. J Pathol. 2015;235(4):646-55.

45. Kalaycio M, Rybicki L, Pohlman B, Sobecks R, Ball E, Cook D, et al. CD8+ T-cell-depleted, matched unrelated donor, allogeneic bone marrow transplantation for advanced AML using busulfan-based preparative regimens. Bone Marrow Transplant. 2005;35(3):247-52.

46. Wikell H, Ponandai-Srinivasan S, Mattsson J, Gertow J, Uhlin M. Cord blood graft composition impacts the clinical outcome of allogeneic stem cell transplantation. Transpl Infect Dis. 2014;16(2):203-12.

47. Naymagon S, Naymagon L, Wong SY, Ko HM, Renteria A, Levine J, et al. Acute graft-versus-host disease of the gut: considerations for the gastroenterologist. Nat Rev Gastroenterol Hepatol. 2017;14(12):711-26.

48. Tang FF, Cheng YF, Xu LP, Zhang XH, Yan CH, Han W, et al. The incidence, risk factors, and outcomes of acute graft-vs-host disease in pediatric T-cell-replete haploidentical hematopoietic stem cell transplantation. Pediatr Transplant. 2020;24(7):e13793.

49. Barba P, Hilden P, Devlin SM, Maloy M, Dierov D, Nieves J, et al. Ex Vivo CD34(+)-Selected T CellDepleted Peripheral Blood Stem Cell Grafts for Allogeneic Hematopoietic Stem Cell Transplantation in Acute Leukemia and Myelodysplastic Syndrome Is Associated with Low Incidence of Acute and Chronic Graft-versus-Host Disease and High Treatment Response. Biol Blood Marrow Transplant. 2017;23(3):452-8. 
50. Faraci M, Caviglia I, Biral E, Morreale G, Giardino S, Garbarino L, et al. Acute graft-versus-host disease in pediatric allogeneic hematopoietic stem cell transplantation. Single-center experience during $10 \mathrm{yr}$. Pediatr Transplant. 2012;16(8):887-93.

51. Chang YJ, Xu LP, Wang Y, Zhang XH, Chen H, Chen YH, et al. Controlled, Randomized, Open-Label Trial of Risk-Stratified Corticosteroid Prevention of Acute Graft-Versus-Host Disease After Haploidentical Transplantation. J Clin Oncol. 2016;34(16):1855-63.

52. Apperley J, Niederwieser D, Huang XJ, Nagler A, Fuchs E, Szer J, et al. Reprint of: Haploidentical Hematopoietic Stem Cell Transplantation: A Global Overview Comparing Asia, the European Union, and the United States. Biol Blood Marrow Transplant. 2016;22(3 Suppl):S15-8.

53. Zhao JY, Liu SN, Xu LP, Zhang XH, Wang Y, Chen YH, et al. Ruxolitinib is an effective salvage treatment for multidrug-resistant graft-versus-host disease after haploidentical allogeneic hematopoietic stem cell transplantation without posttransplant cyclophosphamide. Ann Hematol. 2021;100(1):169-80.

54. Liu SN, Zhang XH, Xu LP, Wang Y, Yan $\mathrm{CH}$, Chen $\mathrm{H}$, et al. Prognostic factors and long-term follow-up of basiliximab for steroid-refractory acute graft-versus-host disease: Updated experience from a large-scale study. Am J Hematol. 2020;95(8):927-36.

55. Shen MZ, Liu XX, Qiu ZY, Xu LP, Zhang XH, Wang Y, et al. Efficacy and safety of mesenchymal stem cells treatment for multidrug-resistant graft-versus-host disease after haploidentical allogeneic hematopoietic stem cell transplantation.[accepted December 17, 2021]. Ther Adv Hematol. 2021.

56. Yan $\mathrm{CH}$, Xu LP, Wang FR, Chen H, Han W, Wang Y, et al. Causes of mortality after haploidentical hematopoietic stem cell transplantation and the comparison with HLA-identical sibling hematopoietic stem cell transplantation. Bone Marrow Transplant. 2016;51(3):391-7.

57. Deng XR, Ren HY, Cen XN, Wang LH, Liang ZY, Wang WS, et al. Effect of graft-versus-host disease on relapse and survival in 100 patients after allogeneic hematopoietic stem cell transplantation. Zhongguo Shi Yan Xue Ye Xue Za Zhi. 2009;17(4):994-8.

58. Hartwell MJ, Özbek U, Holler E, Renteria AS, Major-Monfried H, Reddy P, et al. An early-biomarker algorithm predicts lethal graft-versus-host disease and survival. JCI Insight. 2017;2(3):e89798.

59. Levine JE, Braun TM, Harris AC, Holler E, Taylor A, Miller $\mathrm{H}$, et al. A prognostic score for acute graftversus-host disease based on biomarkers: a multicentre study. Lancet Haematol. 2015;2(1):e21-9.

\section{Tables}

Table 1. Patient characteristics 


\begin{tabular}{|c|c|c|c|}
\hline Characteristics & $\begin{array}{l}\text { Training cohort } \\
(n=335)\end{array}$ & $\begin{array}{l}\text { Validation } \\
\text { cohort } \\
(n=135)\end{array}$ & $P$ value \\
\hline Median age at allo-HSCT, years (range) & $28(1-66)$ & $31(1-64)$ & 0.596 \\
\hline Gender, $n(\%)$ & & & 0.635 \\
\hline Male & $198(59.1)$ & $83(61.5)$ & \\
\hline Female & $137(40.9)$ & $52(38.5)$ & \\
\hline Underlying disease, $n(\%)$ & & & 0.704 \\
\hline Acute myeloid leukemia & $187(55.8)$ & $78(57.8)$ & \\
\hline Acute lymphoblastic leukemia & $143(42.77)$ & $55(40.7)$ & \\
\hline Mixed-phenotype acute leukemia & $5(1.55)$ & $2(1.5)$ & \\
\hline Disease status before allo-HSCT, $n(\%)$ & & & 0.535 \\
\hline CR1 & $321(95.8)$ & $131(97.0)$ & \\
\hline$>$ CR1 & $14(4.2)$ & $4(3.0)$ & \\
\hline Disease risk index before allo-HSCT, $n(\%)$ & & & 0.714 \\
\hline Low and intermediate risk & $268(80.0)$ & $110(81.5)$ & \\
\hline High and very high risk & $67(20.0)$ & $25(18.5)$ & \\
\hline Donor/recipient relation, $n(\%)$ & & & 0.379 \\
\hline Mother donor & $26(7.8)$ & $12(8.9)$ & \\
\hline Collateral donor & $12(3.6)$ & $0(0.0)$ & \\
\hline Others & $297(88.7)$ & $123(91.1)$ & \\
\hline Donor/recipient gender matched, $n(\%)$ & & & 0.258 \\
\hline Female donor/male recipient combination & $57(17.0)$ & $29(21.5)$ & \\
\hline Others & $278(83.0)$ & $106(78.5)$ & \\
\hline HCT-Cl scores before allo-HSCT, $n(\%)$ & & & 0.121 \\
\hline 0 (low-risk) & $237(70.7)$ & $105(77.8)$ & \\
\hline 1-2 (intermediate-risk) & $74(22.1)$ & $23(17.0)$ & \\
\hline$\geq 3$ (high-risk) & $24(7.2)$ & $7(5.2)$ & \\
\hline Median donor age at allo-HSCT, years (range) & $40(9-70)$ & $36(10-63)$ & 0.094 \\
\hline
\end{tabular}


Cytomegalovirus serostatus before HSCT, $n(\%)$

0.501

Donort/recipient+

Donor+/recipient-

Donor-/recipient+

Donor-/recipient-
$312(93.1)$

$11(3.3)$

$10(3.0)$

$2(0.6)$

Number of HLA-A, HLA-B, HLA-DR mismatches, $n(\%)$

1 locu

$\geq 2$ loci

Blood group compatibility, $n(\%)$

Matched

Mismatched

Conditioning regimen, $n(\%)$

Chemotherapy-based regimen

TBI-based regimen

Cell type, median count (range)

MNC counts $\left(\times 10^{8} / \mathrm{kg}\right)$

CD $34+$ cell counts $\left(\times 10^{6} / \mathrm{kg}\right)$

CD $3+$ cell counts $\left(\times 10^{6} \mathrm{~kg}\right)$

CD $4+$ cell counts $\left(\times 10^{6} / \mathrm{kg}\right)$

CD $8+$ cell counts $\left(\times 10^{6} / \mathrm{kg}\right)$

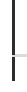

8

$160(47.8)$

CD $14+$ cell counts $\left(\times 10^{6} / \mathrm{kg}\right)$

CD8+/CD3+ cells ratio

CD4+/CD8+ cells ratio

CD4+/CD3+ cells ratio

CD3+/CD14+ cells ratio
$8(2.4)$

327 (97.6)

$175(52.2)$

$325(97.0)$

$10(3.0)$

$9.2(4.4-27.3)$

$3.8(0.7-25.33)$

340.9

(116.2-874.2)

182.5

(68.3-600.1)

126.7

(29.6-347.9)

211.3

(73.3-1065.0)

$0.4(0.2-0.7)$

$1.5(0.4-4.7)$

$0.6(0.2-0.8)$

$1.6(0.6-4.4)$
$128(94.8)$

$3(2.2)$

4 (3.0)

$0(0.0)$
0.914

$3(2.2)$

$132(97.8)$

0.719

$73(54.1)$

$62(45.9)$

0.350

133 (98.5)

2 (1.5)
$9.3(4.2-27.5) \quad 0.218$

$3.9(1.1-29.4) \quad 0.572$

352.0

0.617

(170.4-1172.2)

184.7

0.688

(75.2-492.7)

128.0

0.559

(46.1-1511.2)

215.6

0.373

(95.8-716.9)

$0.4(0.1-1.3)$

0.817

$1.5(0.3-3.0)$

0.672

0.627

$0.5(0.1-0.7)$

0.601


allo-HSCT, allogeneic hematopoietic stem cell transplantation; CR, complete remission; HLA, human leukocyte antigen; $\mathrm{HCTCl}$, hematopoietic cell transplantation-specific comorbidity index; MNC, mononuclear cells; TBI, total body irradiation.

\section{Figures}

\section{Fig. 1}

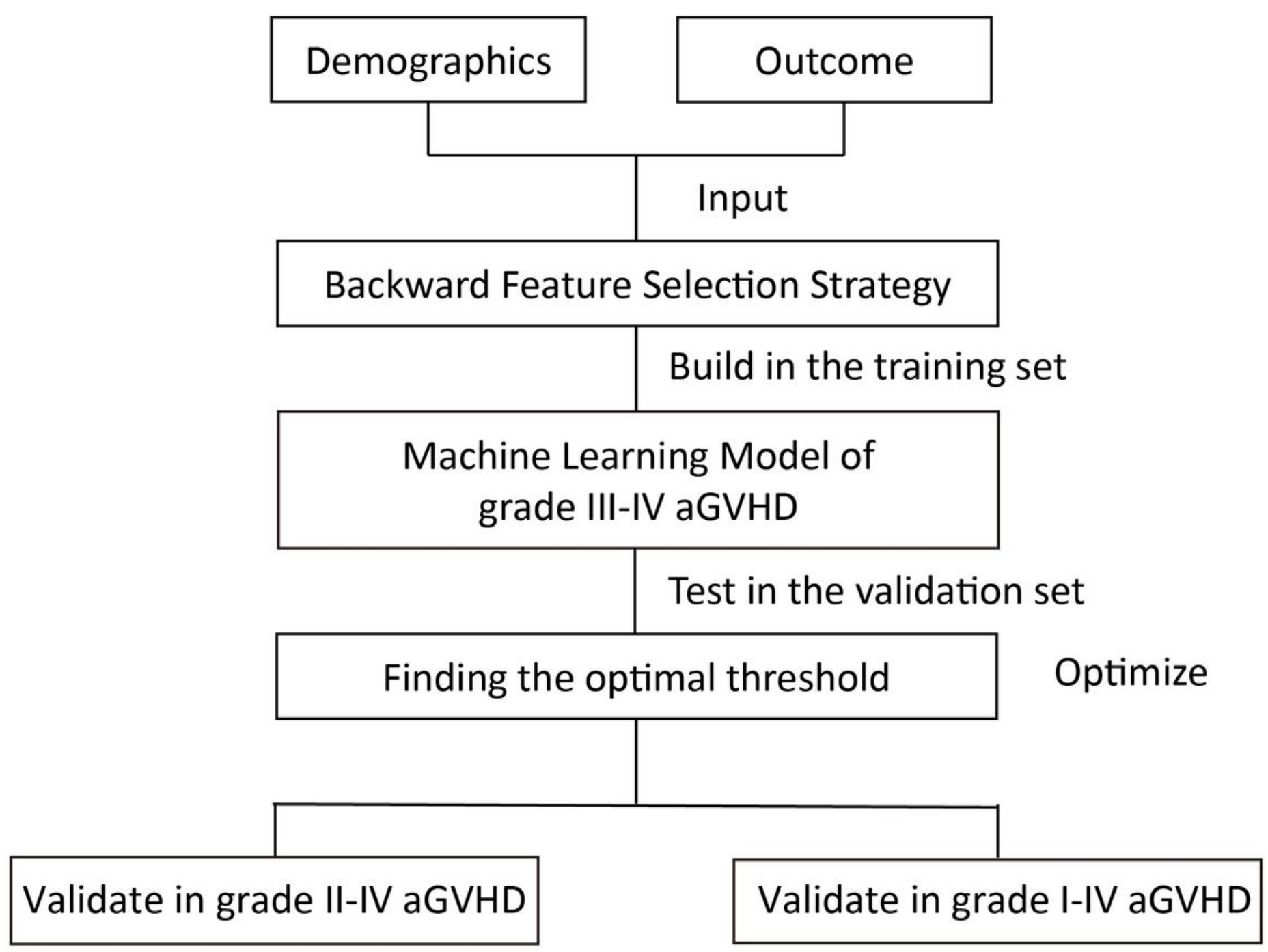

Figure 1

Flow diagram of building machine learning model. 
Fig. 2

(A)
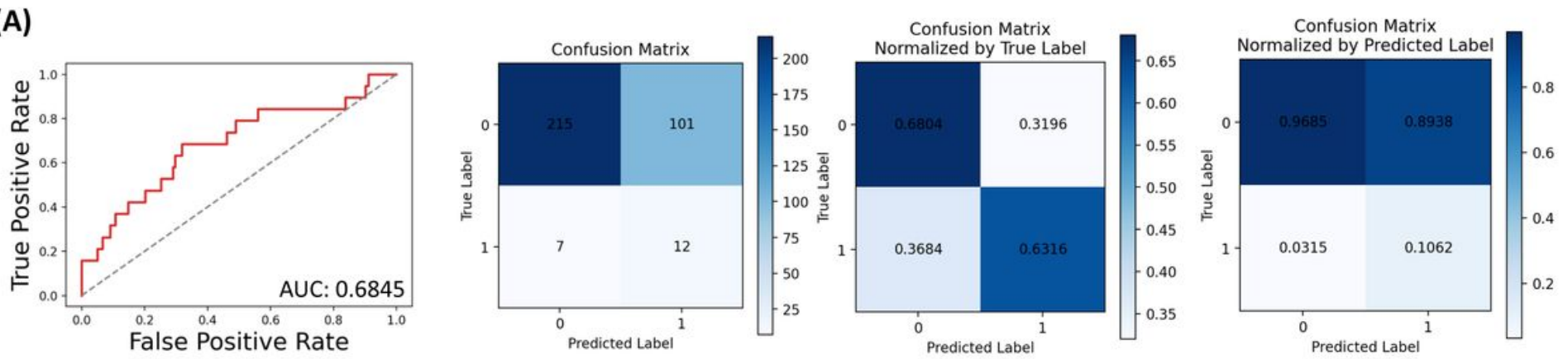

(B)
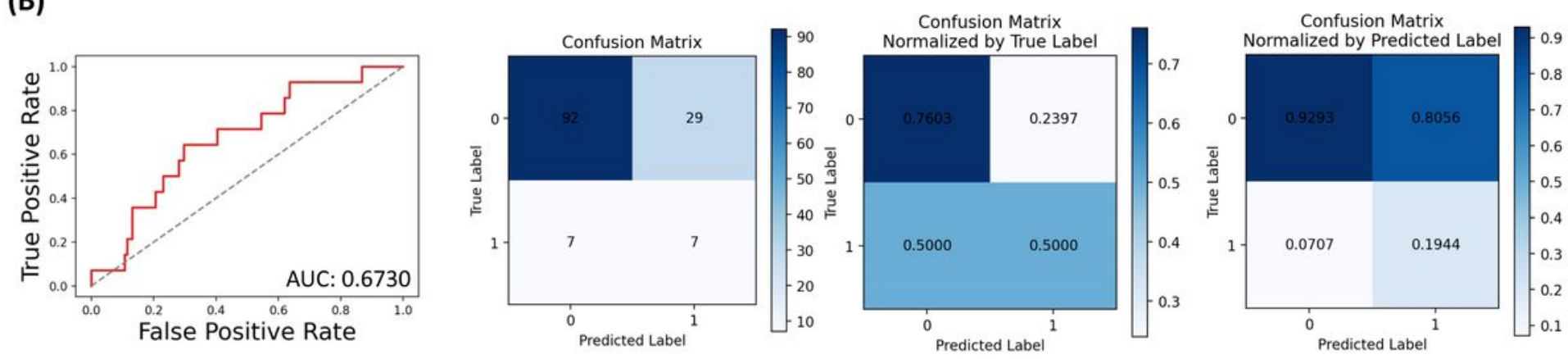

Figure 2

ROC curve and confusion matrix for grade III to IV aGVHD model in the training (A) and validation cohort (B).

Fig. 3
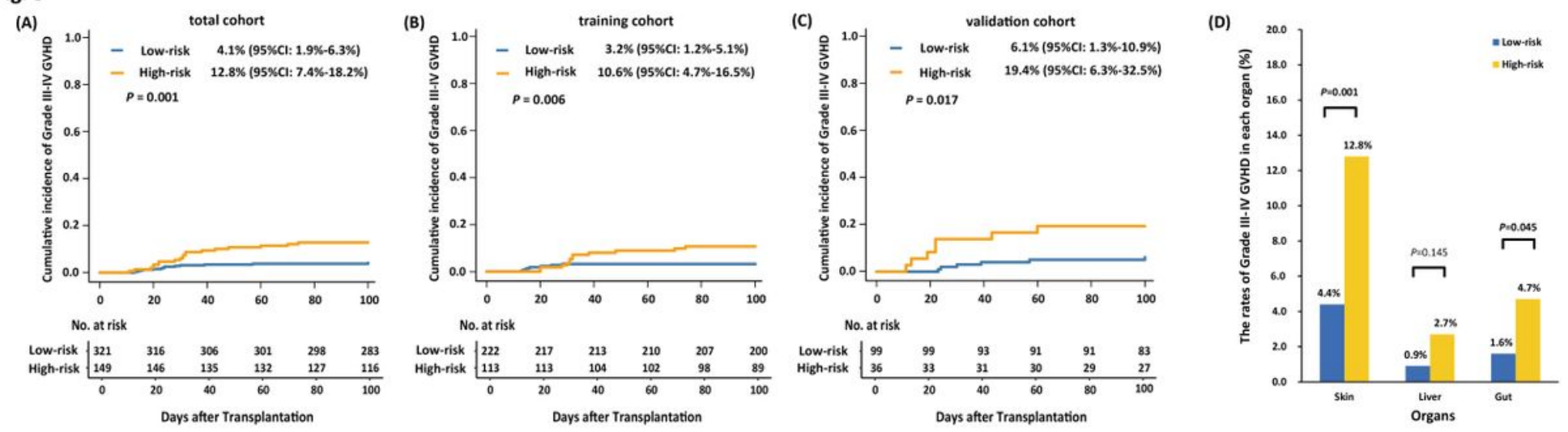

\section{Figure 3}

The 100-day cumulative incidence of grade III to IV aGVHD in the low- and high-risk groups in total (A), training (B), and validation (C) cohort, and (D) the rates of grade III to IV aGVHD of each organ in the lowand high-risk group. 
Fig. 4

(A)

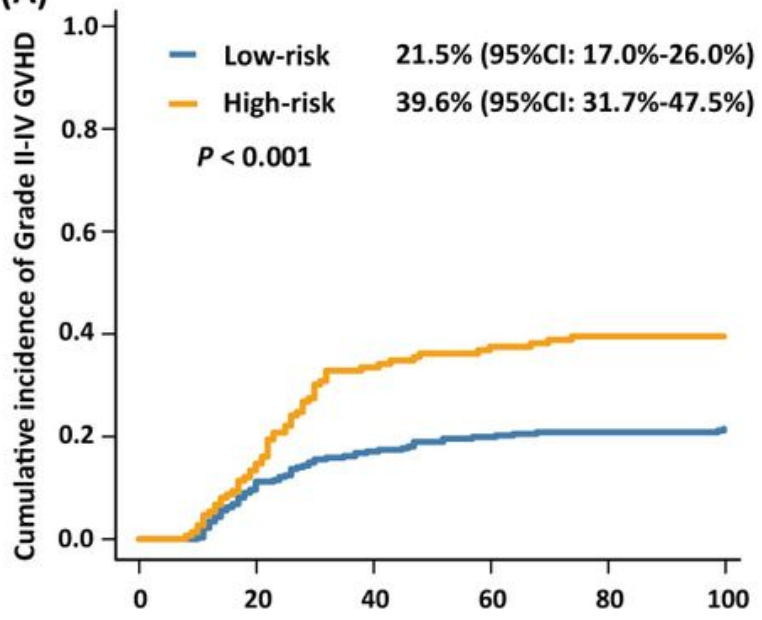

No. at risk

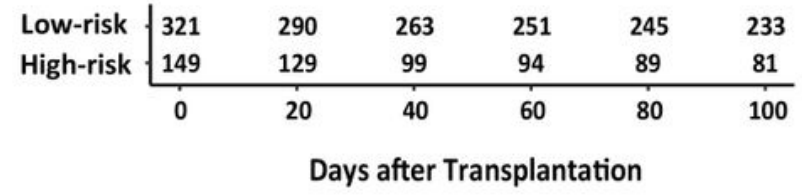

(C)

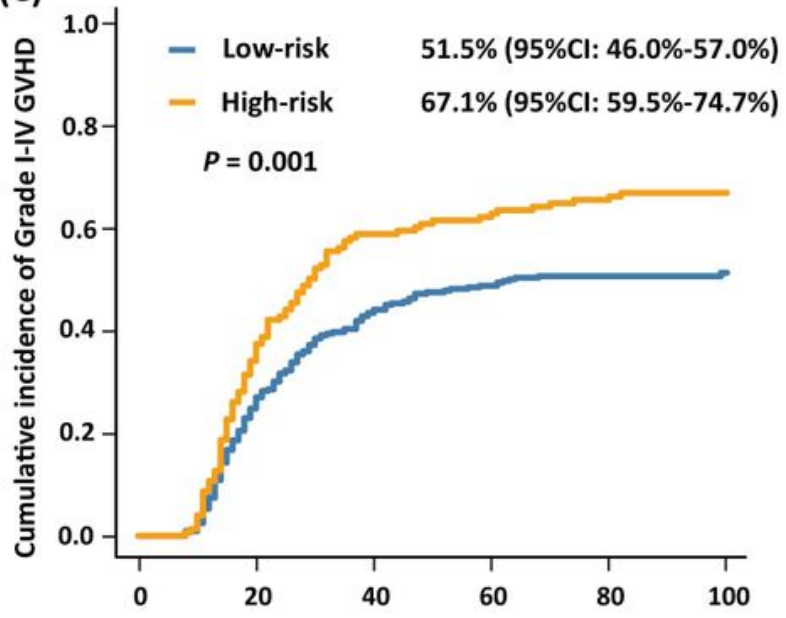

No. at risk

\begin{tabular}{|c|c|c|c|c|c|c|}
\hline Low-risk & 321 & 241 & 178 & 158 & 152 & 143 \\
\hline \multirow[t]{2}{*}{ High-risk } & 149 & 98 & 61 & 56 & 51 & 43 \\
\hline & 0 & 20 & 40 & 60 & 80 & 100 \\
\hline
\end{tabular}

(B)

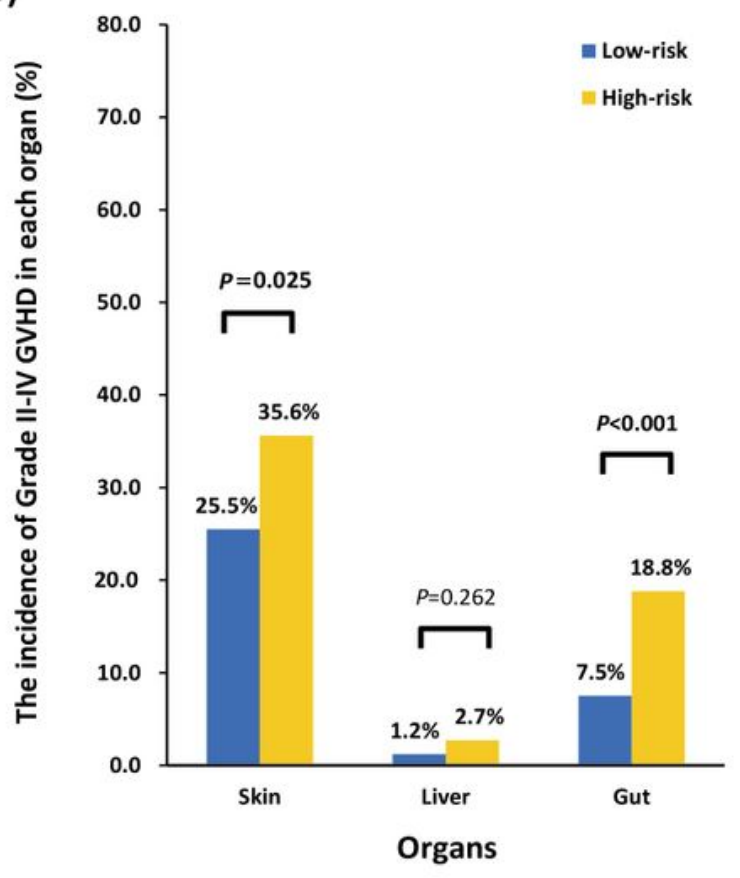

(D)

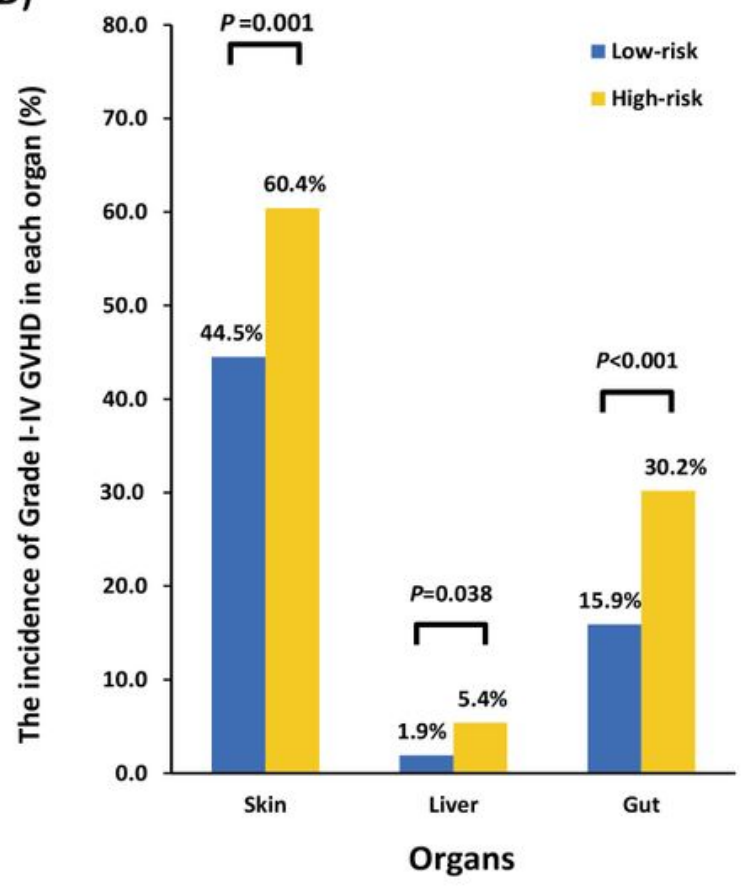

\section{Figure 4}

The association between predicted model and other GVHD endpoint in total population. (A) The 100-day cumulative incidence of grade II to IV aGVHD in the low- and high-risk groups; (B) The rate of grade II to IV aGVHD of each organ in the low- and high-risk groups. (C) The 100-day cumulative incidence of grade I to IV aGVHD in the low- and high-risk groups; (D) The rate of grade I to IV aGVHD of each organ in the lowand high-risk groups. 


\section{Supplementary Files}

This is a list of supplementary files associated with this preprint. Click to download.

- Supplementaryappendix.docx 